\title{
Risk factors for the onset of gallbladder cancer: a review
}

\author{
Zhiyong Tan ${ }^{1}$, Zhuofan Deng², Jianping Gong'*

\begin{abstract}
${ }^{1}$ Department of of Hepatobiliary Surgery, Yunyang County People's Hospital of Chongqing, Chongqing, China ${ }^{2}$ Department of Hepatobiliary Surgery, Second affiliated Hospital of Chongqing Medical University, Chongqing,
\end{abstract} \\ China
}

Received: 03 April2021

Revised: 26 April 2021

Accepted: 30 April 2021

\author{
*Correspondence: \\ Dr. Jianping Gong, \\ E-mail: gongjianping11@126.com
}

Copyright: (c) the author(s), publisher and licensee Medip Academy. This is an open-access article distributed under the terms of the Creative Commons Attribution Non-Commercial License, which permits unrestricted non-commercial use, distribution, and reproduction in any medium, provided the original work is properly cited.

\begin{abstract}
Gallbladder cancer (GBC) is the most common malignancy of the biliary system in clinic, which has the characteristics of insidious onset and high degree of malignancy. Most patients have progressed to an advanced stage when they are diagnosed. Early identification of risk factors of the onset of gallbladder cancer and active intervention are the key to improve the rate of early diagnosis and prognosis of gallbladder cancer. At present, the risk factors related to the onset of gallbladder cancer include gallstone, gallbladder polyps, primary sclerosing cholangitis, etc. In this review, we discuss the relevant latest research on the risk factors of the onset of gallbladder cancer in order to provide clinical evidence for the prevention and early diagnosis of gallbladder cancer. The intervention, follow-up, and monitoring of risk factors should be strengthened, and the possibility of malignancy of the gallbladder should be accurately assessed in combination with factors such as age and sex. In the case of possible malignancy, prophylactic cholecystectomy should be actively performed.
\end{abstract}

Keywords: Gallbladder neoplasms, Morbidity, Risk factors, Review

\section{INTRODUCTION}

Gallbladder cancer (GBC) is a rare malignancy, which ranks sixth in the incidence of gastrointestinal cancer. It is the most common biliary malignancy, accounting for $80 \%$ to $95 \%$ of biliary tract cancer, which is a highly aggressive disease with an extremely poor prognosis, with a median overall survival of 6 months and a 5-year survival of 5\%. ${ }^{1}$ According to the report produced by the International Agency for Research on Cancer (IARC), there are 220000 new cases of GBC worldwide every year and accounting for $1.7 \%$ of all cancer deaths, the burden of gallbladder cancer is expected to increase globally. ${ }^{2}$

GBC has an insidious onset and lack of effective screening measures and specific symptoms, right upper abdominal pain associated with benign biliary diseases may be the first and only symptom. Only a small proportion of patients may have obstructive jaundice secondary to local disease progression and biliary outflow tract obstruction. As a result, most patients are not early diagnosed and prompt treated, the anatomical structures of the gallbladder predispose to early invasion of adjacent structures and lymph nodes by GBC, resulting in loss of the chance of surgical resection and an extremely poor prognosis of patients. A study from Memorial Sloan-Kettering Cancer Center (MSKCC) showed that $36.6 \%$ of GBC patients were at American Joint Committee on Cancer (AJCC) IV stage when diagnosed. ${ }^{3}$

In order to improve the early diagnosis rate of $\mathrm{GBC}$, the priority is to strengthen the early intervention and close follow-up of high-risk individuals. For GBC there remains a paucity of approved preventive and therapeutic options. This may be attributed partly to the etiology and related risk factors of GBC remain insufficiently understood, there are few studies regarding the epidemiology of GBC, and most published studies focused on the surgical treatment 
and related survival rate. Thus, further studies on the risk factors for the initiation of GBC has important clinical significance for its early diagnosis and efficient treatment and ultimately improve the prognosis for these patients, and reduce the societal burden of GBC. In this study, we analyzed the risk factors for the onset of GBC and provided a scientific basis for the prevention and control of GBC.

\section{GBC RISK FACTORS}

\section{Age and sex}

Age

The incidence of GBC rises with age. One study from MSKCC showed the median age of patients was 67 years. ${ }^{3}$ Researchers at the National Cancer Institute found that the incidence of GBC increases steadily with age, the incidence is reported to be highest in populations over 85 years old, the incidence of people aged 18-44 years is much lower than people over 45 years. ${ }^{4}$ Incidental gallbladder cancer (IGBC) refers that the preoperative diagnosis of benign disease but confirmed as GBC by postoperative histopathological diagnosis, which is essentially missed diagnosis and misdiagnosis. A largescale research program in Sweden analysis of the data of patients who accepted cholecystectomy for benign disease showed that patients of IGBC group were older than those in the control group $(70 \pm 11$ vs $54 \pm 16, \mathrm{p}<0.001) .{ }^{5}$ Pitt et al found patients over 65 years were 5.3 times more likely to develop IGBC than patients less than 65 years. $^{3}$

The risk of GBC increases with age, a preoperative careful risk assessment of $\mathrm{GBC}$ before cholecystectomy for elderly patients that matters. This is recommended to prevent the occurrence of incidental GBC to improve outcomes.

\section{$\operatorname{Sex}$}

Worldwide, the incidence rate of GC is three times more common in women than in men. Two nationwide studies conducted in the US all showed that the incidence of gallbladder cancer in women was 1.7 times higher than that in men. ${ }^{4} 7 \mathrm{Wi}$ et al reported that the incidence was similar in men and women in Korea, and there was no significant gender difference in the incidence of gallbladder cancer (the ratio of male to female incidence was 0.96$){ }^{8}$

Swedish study showed that female gender was a preoperative risk factor for incidental gallbladder cancer $(\mathrm{OR}=3.58, \mathrm{p}<0.001){ }^{5}$

GC is one of several cancers that are consistently more prevalent in women worldwide. ${ }^{9}$ It may be closely linked to the reproductive functions and hormone levels of women. Current research has proposed that reproductive and menstrual factors of women are related to the onset of GC.

\section{GALLBLADDER DISEASE}

\section{Gallstone}

Gallstone is a common benign lesion of the gallbladder in clinical practice, and the prevalence of gallstone in adults worldwide was estimated to be $10-20 \%$ worldwide. $^{10}$ Previous study has confirmed that gallstone is one of the most important risk factors for GBC, and gallstone is strongly correlated with the onset of GBC.

Hsing et al reported a large-scale case-control study in Shanghai, $83.7 \%$ of patients with GBC have a history of gallstone. ${ }^{11}$ Patients with gallstone without a family history of gallstone had a 21-fold higher risk of GBC than healthy controls, and those with gallstone and a family history of gallstone had a 57-fold higher risk, and only $0.5 \%$ to $3 \%$ of patients with gallstone will develop GBC. Cariati et al conducted a multicenter study included 75 patients with GBC, found that $96 \%$ of patients had gallstone. $^{12}$

A close link between the size of gallstone and the onset of GBC was confirmed in decades, especially gallstone larger than $3 \mathrm{~cm}$ in diameter. It takes time for gallstone to become larger. Jain et al found a causal relationship between longterm gallstone and GBC. ${ }^{13}$

Overall, gallstone is indeed a significant risk factor for $\mathrm{GBC}$, the risk of $\mathrm{GBC}$ rises with the increase in the diameter and course. The size of gallstone may mark the course of gallstone and chronic inflammation in the gallbladder, and is ultimately linked to the onset of GBC. Studies on the relationship between gallstone and GBC will help to find the best way to achieve secondary prevention of GBC by preventive cholecystectomy in high risk patients (e.g. $>3 \mathrm{~cm}$ in diameter or $>15$ years in disease course). ${ }^{14}$

\section{Gallbladder polyps}

Gallbladder polyps are elevations of the gallbladder wall that project into the lumen. Gallbladder polyps are estimated to have a prevalence of approximately $5 \%$ in the global population, with $5 \%$ of gallbladder polyps are malignant or have malignant potential. 2017 European Multi-Society Joint Guidelines pointed out that important risk factors of GBC include gallbladder polyp diameter $>10 \mathrm{~mm}$, sessile polyps, and gallbladder polyps increase $\geq 2 \mathrm{~mm}$ in the diameter during follow-up, but the evidence is mostly from retrospective studies, and the quality of evidence is low. ${ }^{15}$

A 20-year cohort study showed that polyps less than 10 $\mathrm{mm}$ have a low correlation with GBC, during the followup of 1549 person-years in the group of 507 patients whose polyps are increasing over $10 \mathrm{~mm}$ in size, no one was 
diagnosed with GBC. ${ }^{16}$ It seems that the increase in the size of gallbladder polyps also did not appear to be associated with later GBC. Fujiwara et al reported that gallbladder polyp size was an important risk factor for $\mathrm{GBC}$, and the positive predictive values of diameter $\geq 10$ $\mathrm{mm}, \geq 15 \mathrm{~mm}$, and $\geq 20 \mathrm{~mm}$ were $16.4 \%, 55.9 \%$, and $94.1 \%$, respectively, and sessile polyps was significant appear in malignant lesions than in benign lesions (60\% vs $3.4 \%, \mathrm{p}<0.00001) .{ }^{17} \mathrm{~A}$ single-centre, retrospective cohort study from China found that the presence of polyp growth was associated with premalignancy $(\mathrm{OR}=5.366,95 \% \mathrm{CI}$ 1.466-19.637, $\mathrm{p}=0.011) .{ }^{18} \mathrm{~A}$ systematic review including 43 studies concluded that diameter of gallbladder polyps $\geq 10 \mathrm{~mm}$ is appropriate as a recommended cut-off point for cholecystectomy, with twenty studies contained data of 228 malignant polyps, of which 29 were 5 to $10 \mathrm{~mm}$ in diameter, but none were smaller than $5 \mathrm{~mm} .{ }^{19}$

The standard of $10 \mathrm{~mm}$ may be the most important factor in decision making for surgery in clinical practice. However, this cut-off was likely to miss a large number of true gallbladder polyps below the threshold, and cholecystectomy will be performed in patients with pseudopolyps larger than $10 \mathrm{~mm}$. Cholecystectomy is recommended for polypoid lesion of the gallbladder greater than or equal to $10 \mathrm{~mm}$ or during follow-up gallbladder polyp increases by $2 \mathrm{~mm}$ or more. ${ }^{15}$ But the most recent report found that the increase in the size of gallbladder polyps did not appear to be associated with later GBC, which provides new ideas in Intervention of gallbladder polyps. ${ }^{16}$ Patel et al found that the management of gallbladder polyps based on the European consensus guidelines would be clinically cost-effective, but will significantly increase the proportion of patients requiring cholecystectomy. ${ }^{15,20}$ Currently, no studies have been conducted to assess the impact of following these guidelines, larger-scale retrospective and prospective studies are needed to assess the benefit of managing gallbladder polyps in accordance with current guidelines.

In order to obtain the greatest clinical and economic benefits, the correlation between the size and growth of gallbladder polyps and GBC should be further studied, the best indications for preventive cholecystectomy for gallbladder polyps should be carefully considered, and choose the best management strategy for gallbladder polyps.

\section{Gallbladder adenomyomatosis}

Gallbladder adenomyomatosis (GA) is a proliferative disease characterized by epithelial proliferation and hypertrophy of the muscles of the gallbladder wall with an outpouching of the mucosa into or through the thickened muscular layer, this is, the rokitansky-aschoff sinuses (RAS). There are three macroscopic forms of GA: segmental form $(>60 \%)$, fundal form $(30 \%)$ and diffuse form $(<5 \%)$. GA has traditionally been ascribed to have no malignant potential and its associations with early GBC remains unclear.
Kim et al retrospective analysis of 113 GA cases, no GBC was found. Indeed, there is recent evidence that GA is not always benign. ${ }^{21,22}$. Both segmental form and fundal form were found to be risk factors for onset of GBC by Morikawa et al. ${ }^{23}$

Currently, whether GA is a risk factor for GBC is in debate. In fact, despite regular follow-up, quite part of patients with GA and GBC are found at an advanced stage of GBC. In summary, based on the study results, no conclusion can be made about whether GA need for preventive cholecystectomy, asymptomatic GA can be followed up for observation, while surgery when combined with other risk factors for $\mathrm{GBC}$ is not controversial.

\section{BILE DUCT DISEASE}

\section{Primary sclerosing cholangitis}

Primary sclerosing cholangitis (PSC) is a rare, chronic cholestatic liver disease characterised by intrahepatic or extrahepatic stricturing, or both, with bile duct fibrosis. its incidence is increasing in recent years. Patients with PSC have a significantly increased risk of various abdominal malignancies, and tumors account for $40-50 \%$ of the total causes of death in patients with PSC. ${ }^{24} \mathrm{PSC}$ is an important risk factor for GBC, a lifetime risk of patients with PSC is estimated to be $3-14 \% .^{25}$

Studies from Swedish and Cleveland clinic report that the prevalence of GBC among PSC patients was, respectively $2.5 \%$ and $3.5 \% .^{26,27}$ Rabiee et al reported that patients with PSC increase 9-fold risk of GBC than normal human, they should have regular abdominal ultrasound examinations, and cholecystectomy should be performed if the size of gallbladder polyps is found to be greater than $8 \mathrm{~mm} .^{28}$

Patients with PSC are more likely to have malignant gallbladder polyps, even the polyps are smaller than 10 $\mathrm{mm}$. There have been no reports of predictors of malignancy in gallbladder mass or polyps in PSC patients. Current PSC guidelines recommend immediate cholecystectomy for gallbladder polyps of any size. ${ }^{29}$ However, recent study has shown that it is reasonable for PSC patients with gallbladder polyps to undergo shortterm ultrasound monitoring before immediate cholecystectomy in the absence of high-risk imaging features or severe clinical problems. ${ }^{30}$

Cancer is the main cause of death in patients with PSC. Next, increased attention should be paid to the gallbladder of PSC patients, regular monitoring and early treatment of gallbladder disease should be emphasized.

\section{Pancreatobiliary maljunction}

Pancreaticobiliary maljunction (PBM) is a congenital malformation in which the pancreatic and bile ducts join anatomically at a high position outside the duodenal wall, 
forming an abnormally long common channel. Sphincter of oddi is affected, resulting pancreatic juice reflux into the biliary tract, ultimately leading to pathological conditions of the pancreas and biliary tract. There are two types of PBM, with biliary dilatation (congenital biliary dilatation) and without biliary dilatation. PBM is considered an independent risk factor for the onset of GBC.

Muraki et al found that $8 \%$ of patients with GBC in the US had PBM (24/300), in Japan the situation is similar $(15 / 171,8.8 \%)$, but PBM is usually not diagnosed initially. ${ }^{31}$ Hyvarinen et al reported that the estimated prevalence of PBM among patients with GBC was $17 \% .{ }^{32}$ Fujimoto et al observed that the incidence of GBC among PBM patients was 58\% (7/12), and elevated bile amylase levels in PBM patients with serum amylase levels close to the upper limit of normal range seems may be related with GBC. $^{33}$

Current study showed that activated proteolytic enzymes and other cytotoxic substances continuously damage the biliary epithelium of patients with PBM, and the resulting chronic inflammation leads to repeated circulation of biliary epithelial injury and healing, leading to mutations in oncogenes and/or tumor suppressor genes in epithelial cells, which leads to the occurrence of biliary tract cancer. ${ }^{34}$ Given the high correlation between PBM and biliary tract cancer (mainly GBC), Cholecystectomy and monitoring of the bile duct are necessary after PBM diagnosis was made.

\section{INFLAMMATORY AND INFECTIOUS FACTORS}

\section{Chronic cholecystitis, porcelain gallbladder}

Chronic cholecystitis is a risk factor for the onset of GBC. Chronic inflammation can lead to abnormal deposition of calcium on the gallbladder wall causing calcification of the gallbladder wall, which is customarily called porcelain gallbladder. By the distribution of calcifications between the gallbladder wall, it can be divided into selective mucosal subtypes and complete intramural subtypes.

For decades, management of porcelain gallbladder has been controversial. Porcelain gallbladder has traditionally been considered to have a close link with the occurrence of GBC and requires prophylactic cholecystectomy. Newer evidence suggests a much lesser association.

A systematic reviews showed that the incidence of GBC in the porcelain gallbladder group was $6 \%$ and higher than the control group (1\%). ${ }^{35}$ Chen et al found that porcelain gallbladder is not related to the increased risk of GBC but to the high risk of complications after cholecystectomy, so cholecystectomy is not suitable for asymptomatic porcelain gallbladder patients. ${ }^{36}$ Des et al found that while there was no significant difference in failure rates between surgery and short-term observation, prophylactic cholecystectomy for each patient with porcelain gallbladder does not seem reasonable. ${ }^{37}$
Despite lacking of evidence-based guidelines, many people still regard porcelain gallbladder as the indication of cholecystectomy. The above studies have shown that the link between porcelain gallbladder and GBC is not as high as traditionally expected. It seems not be reasonable to perform cholecystectomy for all patients with porcelain gallbladder. A regularly monitoring of gallbladder wall may be more reasonable for most patients. More studies are needed to evaluate the correlation between the two and make clinical decisions on management of porcelain gallbladder based on evidence-based medical.

\section{Special bacterial infections}

Some special types of bacterial infections are also risk factors for the onset of GBC, S. typhi (Salmonella typhi) and $\mathrm{H}$. pylori (Helocobacter pylori) Infection have been reported. Koshiol et al found S. typhi carriers have a 12fold increased risk of GBC. ${ }^{38}$ Their meta-analysis of more than 1,000 cases of GBC indicated that the total relative risk of GBC in patients with positive S. typhi vi antibody was 4.6.

It has been confirmed that chronic colonization infection and the injury of the gallbladder caused by biofilms and typhoid toxins produced by S. typhi may be key factors in the onset of GBC. ${ }^{39}$

Hassan et al found there were significant differences in mucosal hyperplasia, metaplasia and lymphocyte infiltration between the histological findings of H. pyloriinfected gallbladder and non-infected ones ( $p$ values of $0.028,0.049$ and 0.022 , respectively). ${ }^{40} \mathrm{H}$. pylori infection in aggravating the mucosal lesions (mucosal hyperplasia, metaplasia, and lymphoid infiltration) of the gallbladder that is considered potentially precancerous.

Special types of bacterial infections are risk factor for the onset of GBC, which may be linked to the chronic inflammatory state and the gallbladder injury caused by substances produced by bacteria, and further mechanisms need to be explored.

It may be of special significance to search for markers of two special bacteria in the serum or bile of high-risk groups with GBC.

\section{OTHER RISK FACTORS}

\section{Reproductive/menstrual factors}

Jackson et al pooled data of more than 1.5 million women, found that high parity was associated with risk of GBC (HR $\geq 5$ vs 0 births $1.72 ; 95 \%$ CI 1.25-2.38), age at menarche (HR per year increase 1.15; 95\% CI 1.06-1.24) was associated with GBC risk in Asian women while reproductive years were associated with GBC risk (HR per 5 years 1.13 ; $95 \%$ CI 1.04-1.22) in non-Asian women. ${ }^{41}$ In Shanghai, higher parity, younger age at first birth and older age at menarche on GBC risk were more pronounced 
among women with gallstone. ${ }^{42}$ A prospective study ${ }^{43}$ suggests that women with irregular or longer cycles may have an increased risk for GBC and female hormones may play an important role in the etiology of GBC.

These associations suggest that female hormones may play an important role in the onset of GBC. Progesterone have been suggested to reduce the risk of ovarian cancer. Estrogen contributes to the formation of gallstone by elevating biliary cholesterol, but the roles of progesterone and estrogen in GBC etiology are unclear. Saranga et al found progesterone receptor and estrogen receptor were found to be expressed simultaneously in most of the GBC and sex hormone receptor positive GBC Patients had significantly more metaplasia and dysplasia in the gallbladder. ${ }^{44}$

Reproductive/menstrual factors in women are associated with the risk of GBC, while the role of sex hormones in the onset of $\mathrm{GBC}$ is unclear. In future, deep studies on sex hormone receptors in female patients with GBC may find the role of sex hormones in GBC.

\section{Obesity}

Obesity and the resulting metabolic syndrome have been thought to be associated with an increased risk of GBC. About one-third of GBCs worldwide result from obesity. ${ }^{45}$ A large population cohort study demonstrated that each 5 $\mathrm{kg} / \mathrm{m}^{2}$ increase in BMI was roughly linearly associated with GBC (hazard ratio $=1.31$, CI 1.12-1.52; $\mathrm{p}<0.0001) .{ }^{46}$ A meta-analysis found that compared with 'normal' weight, the summary RR of GBC were 1.14 for overweight individuals (BMI 25-30 $\mathrm{kg} / \mathrm{m}^{2}$ ) and 1.56 for obese individuals $\left(\right.$ BMI $\left.>30 \mathrm{~kg} / \mathrm{m}^{2}\right){ }^{47}$ Other studies also suggest that excess body weight is associated with a significantly increased risk of GBC. ${ }^{48,49}$

The above results confirmed that overweight and obesity are closely related to the onset of GBC, especially in the female population. Obesity is an important but preventable risk factor for $\mathrm{GBC}$, reducing excess weight can directly reduce the risk of GBC. Emphasizing the importance of weight control education in the high-risk population with GBC.

\section{Geography/race}

The incidence of GBC has a wide range of geographical and ethnic differences. It is very high in Latin America, East Asia, and South Asia, while it is low in the United States and most Western European countries. ${ }^{50}$ The incidence of GBC was highest in Chile $(27 / 100,000)$, followed by northern India $(21.5 / 100,000)$; other regions included Poland (14/100,000), southern Pakistan $(11.3 / 100,000)$, and Japan $(7 / 100,000){ }^{2}$ In the United States, the incidence of GBC is only $1.56 / 100,000$ personyears. ${ }^{4}$ Native Americans often troubled by GBC, and Mapuche Indians in South America have the highest prevalence of GBC worldwide: 12.3 per 100,000 in men and 27.3 per 100,000 in women. ${ }^{1}$ Henley et al reported that in US, the incidence of GBC in Indians and Alaska natives is three times higher than in non-Latino whites. ${ }^{51}$ Van et al found that in US, the incidence of GBC was highest in the Hispanic population (7.4/100,000 person-years), while the overall incidence in Americans is only 1.56/100,000 person-years. ${ }^{4}$ Bermejo et al found that each $1 \%$ increase in the Mapuche proportion represented a $3.7 \%$ increased mortality risk by GBC $\left(95 \%\right.$, CI $\left.3.1-4.3 \%, P=6 \times 10^{-27}\right)$, indicating a close link between ethnic ancestry and GBC risk. ${ }^{52}$ Jackson et al found women of Mapuche ethnicity have higher levels of the inflammatory marker IL-8 than non-Mapuche women, we can carry out more in-depth research to clarify its role in gallbladder disease to elucidate ethnic differences in gallstone and GBC among American natives. ${ }^{53}$ A study conducted in the UK by Aldouri et al showed that among 5391 patients who underwent cholecystectomy, those of Indian ancestry had an almost 13-fold higher risk of developing GBC than Caucasians. ${ }^{54}$

GBC has significant geographical and ethnic specificity, which is closely related to the economic, social, environmental and public health levels between different regions. At the same time, it is related to the genetic factors between different races. There may be direct or indirect genetic susceptibility factors of GBC among natives in some areas. GBC requires the development of preventive strategies adapted to local conditions.

\section{Dietary habits}

A cohort study in Japan with a follow-up of 15 years showed that the risk of GBC was positively correlated with the amount of smoking, and drinking alcohol increased the risk of GBC in men. ${ }^{55} \mathrm{~A}$ recent meta-analysis found that smokers had a higher risk of GBC compared with nonsmokers $(\mathrm{RR}=1.33,95 \% \mathrm{CI} 1.17-1.51) .{ }^{56}$ The risk of GBC was linearly correlated with smoking intensity and smoking duration, and the risk of GBC in smokers after quitting smoking did not decrease significantly with increasing smoking cessation time. Certainly, further work is needed to elaborate the relationship between smoking and alcohol consumption and the risk of GBC.

A large-scale prospective cohort study in Japan showed that no risk reduction was found for GBC with vegetable and fruit consumption. ${ }^{57}$ A prospective study showed that adherence to a healthy dietary pattern (mainly including the DASH diet and the Mediterranean diet) was negatively correlated with the incidence of GBC, suggesting that these two dietary patterns may play a role in the primary prevention of GBC. ${ }^{58}$

\section{Aflatoxin exposure}

Aflatoxin is a mycotoxin produced by the fungus Aspergillus, and aflatoxin exposure is mainly associated with human consumption of food contaminated with aflatoxin. The WHO has classified aflatoxin as a class I 
carcinogen in 1993, and there is a positive correlation between aflatoxin and liver cancer, particularly playing a synergistic role with chronic hepatitis B virus infection in the development of hepatocellular carcinoma. A study in Chile showed that adducts formed by aflatoxin and albumin were more likely to be detected in the peripheral blood of GBC patients, OR=9.4 (compared with gallstone control group); $\mathrm{OR}=13.2$ (compared with community control group). ${ }^{59}$ The association between exposure to aflatoxin and GBC was statistically significant. On the basis of this study, a larger scale study was conducted in Shanghai, similarly, showed a strong association between aflatoxin exposure and GBC. ${ }^{60}$

Aflatoxin is likely to be an important risk factor for GBC. Recently, studies have been conducted to explore the relationship between aflatoxin exposure and the onset of GBC. In parallel, more studies are needed in this area.

\section{CONCLUSION}

In conclusion, the onset of GBC is related to a variety of risk factors. The intervention, follow-up, and monitoring of related biliary tract diseases should be strengthened, and the possibility of malignancy of the gallbladder should be accurately assessed in combination with factors such as age and sex. In the case of possible malignancy, prophylactic cholecystectomy should be actively performed. This review has not included the risk factors at the molecular biological level, such as biomarkers and genotypes of gallbladder, which will have important clinical application value in the future. There are so few identified risk factors for $\mathrm{GBC}$, more large-sample, multicenter, prospective studies are needed to further explore the controversial risk factors. Based on trusted work, an effective risk assessment model of GBC will greatly optimize the management of the high-risk population, provide opportunities for the prevention, early diagnosis and early treatment of this disease. Finally reduce the burden of GBC to an extent.

\section{Funding: No funding sources}

Conflict of interest: None declared

Ethical approval: Not required

\section{REFERENCES}

1. Hundal R, Shaffer EA. Gallbladder cancer: epidemiology and outcome. Clin Epidemiol. 2014;6:99-109.

2. Bray F, Ferlay J, Soerjomataram I, Siegel RL, Torre LA, Jemal A. Global cancer statistics 2018: Globocan estimates of incidence and mortality worldwide for 36 cancers in 185 countries. CA Cancer J Clin. 2018;68(6):394-424.

3. Duffy A, Capanu M, Abou GK, Huitzil D, Jarnagin W, Fong Y, Angelica M, et al. Gallbladder cancer (GBC): 10-year experience at Memorial SloanKettering Cancer Centre (MSKCC). J Surg Oncol. 2008;98(7):485-9.
4. Van DAL, Shiels MS, Jones GS, Pfeiffer RM, Petrick JL, Beebe JL, Koshiol J. Biliary tract cancer incidence and trends in the United States by demographic group, 1999-2013. Cancer. 2019;125(9):1489-98.

5. Muszynska C, Lundgren L, Lindell G, Andersson R, Nilsson J, Sandstrom P, Andersson B. Predictors of incidental gallbladder cancer in patients undergoing cholecystectomy for benign gallbladder disease: Results from a population-based gallstone surgery registry. Surgery. 2017;162(2):256-63.

6. Pitt SC, Jin LX, Hall BL, Strasberg SM, Pitt HA. Incidental gallbladder cancer at cholecystectomy: when should the surgeon be suspicious? Ann Surg. 2014;260(1):128-33.

7. Rahman R, Simoes EJ, Schmaltz C, Jackson CS, Ibdah JA. Trend analysis and survival of primary gallbladder cancer in the United States: a 1973-2009 population-based study. Cancer Med. 2017;6(4):87480.

8. Wi Y, Woo H, Won YJ, Jang JY, Shin A. Trends in Gallbladder Cancer Incidence and Survival in Korea. Cancer Res Treat. 2018;50(4):1444-51.

9. Randi G, Franceschi S, La Vecchia C. Gallbladder cancer worldwide: geographical distribution and risk factors. Int J Cancer. 2006;118(7):1591-602.

10. Lammert F, Gurusamy K, Ko CW, Miquel JF, Mendez SN, Portincasa P, et al. Gallstones. Nat Rev Dis Primers. 2016;2:16024.

11. Hsing AW, Bai Y, Andreotti G, Rashid A, Deng J, Chen J, Goldstein AM, et al. Family history of gallstones and the risk of biliary tract cancer and gallstones: a population-based study in Shanghai, China. Int J Cancer. 2007;121(4):832-8.

12. Cariati A, Piromalli E, Cetta F. Gallbladder cancers: associated conditions, histological types, prognosis, and prevention. Eur J Gastroenterol Hepatol. 2014;26(5):562-9.

13. Jain K, Mohapatra T, Das P, Misra MC, Gupta SD, Ghosh M, et al. Sequential occurrence of preneoplastic lesions and accumulation of loss of heterozygosity in patients with gallbladder stones suggest causal association with gallbladder cancer. Ann Surg. 2014;260(6):1073-80.

14. Andrea C, Enzo A. Cholesterol Gallstones Larger Than $3 \mathrm{~cm}$ Appear to Be Associated With Gallbladder Cancer: Identification of a High Risk Group of Patients That Could Benefit From Preventive Cholecystectomy. Ann Surg. 2016;263(3):56.

15. Wiles R, Thoeni RF, Barbu ST, Vashist YK, Rafaelsen SR, Dewhurst C, et al. Management and follow-up of gallbladder polyps: Joint guidelines between the European Society of Gastrointestinal and Abdominal Radiology (ESGAR), European Association for Endoscopic Surgery and other Interventional Techniques (EAES), International Society of Digestive Surgery - European Federation (EFISDS) and European Society of Gastrointestinal Endoscopy (ESGE). Eur Radiol. 2017;27(9):385666. 
16. Szpakowski JL, Tucker LY. Outcomes of Gallbladder Polyps and Their Association With Gallbladder Cancer in a 20-Year Cohort. JAMA Netw Open. 2020;3(5):205143.

17. Fujiwara K, Abe A, Masatsugu T, Hirano T, Sada M. Effect of gallbladder polyp size on the prediction and detection of gallbladder cancer. Surg Endosc. 2020.

18. Wu T, Sun Z, Jiang Y, Yu J, Chang C, Dong X, Yan S. Strategy for discriminating cholesterol and premalignancy in polypoid lesions of the gallbladder: a single-centre, retrospective cohort study. ANZ J Surg. 2019;89(4):388-92.

19. Babu BI, Dennison AR, Garcea G. Management and diagnosis of gallbladder polyps: a systematic review. Langenbecks Arch Surg. 2015;400(4):455-62.

20. Patel K, Dajani K, Vickramarajah S, Huguet E. Five year experience of gallbladder polyp surveillance and cost effective analysis against new European consensus guidelines. HPB. 2019;21(5):636-42.

21. Kim JH, Jeong IH, Han JH, Kim JH, Hwang JC, Yoo $\mathrm{BM}$, et al. Clinical/pathological analysis of gallbladder adenomyomatosis; type and pathogenesis. Hepatogastroenterology. 2010;57(99):420-5.

22. Howe L, Kowdley GC, Cunningham SC. Gallbladder adenomyomatosis: not always benign. HPB. 2017;19(6):557.

23. Morikawa T, Okabayashi T, Shima Y, Sumiyoshi T, Kozuki A, Saisaka Y, et al. Adenomyomatosis Concomitant with Primary Gallbladder Carcinoma. Acta Med Okayama. 2017;71(2):113-8.

24. Fung BM, Lindor KD, Tabibian JH. Cancer risk in primary sclerosing cholangitis: Epidemiology, prevention, and surveillance strategies. World J Gastroenterol. 2019;25(6):659-71.

25. Razumilava N, Gores GJ, Lindor KD. Cancer surveillance in patients with primary sclerosing cholangitis. Hepatology. 2011;54(5):1842-52.

26. Said K, Glaumann H, Bergquist A. Gallbladder disease in patients with primary sclerosing cholangitis. J Hepatology. 2008;48(4):598-605.

27. Torabi SB, Edwards K, Shen B. Frequency, Risk Factors, and Outcome of Gallbladder Polyps in Patients With Primary Sclerosing Cholangitis: A Case-Control Study. Hepatol Commun. 2018;2(12):1440-5.

28. Rabiee A, Levy C. Medical management of primary sclerosing cholangitis. Clin Liver Dis. 2014;3(3):4851.

29. Tabibian JH, Ali AH, Lindor KD. Primary Sclerosing Cholangitis, Part 2: Cancer Risk, Prevention, and Surveillance. Gastroenterol Hepatol. 2018;14(7):427-32.

30. Erp LW, Cunningham M, Narasimman M, Ale AH, Jhaveri K, Drenth JPH, et al. Risk of gallbladder cancer in patients with primary sclerosing cholangitis and radiographically detected gallbladder polyps. Liver Int. 2020;40(2):382-92.

31. Muraki T, Pehlivanoglu B, Memis B, Reid MD, Uehara T, Basturk $\mathrm{O}$, et al. Pancreatobiliary
Maljunction-Associated Gallbladder Cancer is as Common in the West, Shows Distinct Clinicopathologic Characteristics and Offers an Invaluable Model for Anatomy-Induced RefluxAssociated Physio-Chemical Carcinogenesis. Ann Surg. 2020.

32. Hyvarinen I, Hukkinen M, Kivisaari R, Parviainen H, Nordin A, Pakarinen MP. Increased prevalence of pancreaticobiliary maljunction in biliary malignancies. Scand J Surg. 2019;108(4):285-90.

33. Fujimoto T, Ohtsuka T, Nakashima Y, Gotoh Y, Date $\mathrm{K}$, Mori Y, et al. Elevated bile amylase level without pancreaticobiliary maljunction is a risk factor for gallbladder carcinoma. J Hepatobiliary Pancreat Sci. 2017;24(2):103-8.

34. Kamisawa T, Kuruma S, Chiba K, Tabata T, Koizumi $\mathrm{S}$, Kikuyama M. Biliary carcinogenesis in pancreaticobiliary maljunction. J Gastroenterol. 2017;52(2):158-63.

35. Schnelldorfer T. Porcelain gallbladder: a benign process or concern for malignancy?. J Gastrointest Surg. 2013;17(6):1161-8.

36. Chen GL, Akmal Y, Fronzo AL, Vuong B, Connor V. Porcelain Gallbladder: No Longer an Indication for Prophylactic Cholecystectomy. Am Surg. 2015;81(10):936-40.

37. Des JH, Duy L, Scheirey C, Schnelldorfer T. Porcelain Gallbladder: Is Observation a Safe Option in Select Populations?. J Am Coll Surg. 2018;226(6):1064-9.

38. Koshiol J, Wozniak A, Cook P, Adaniel C, Acevedo J, Azocar L, et al. Salmonella enterica serovar Typhi and gallbladder cancer: a case-control study and meta-analysis. Cancer Med. 2016;5(11):3325-310.

39. Di DEG, Cavallo I, Pontone M, Toma L, Ensoli F. Biofilm Producing Salmonella Typhi: Chronic Colonization and Development of Gallbladder Cancer. Int J Mol Sci. 2017;18(9):1887.

40. Hassan EH, Gerges SS, Atrebi KA, Bassyouni HT. The role of $\mathrm{H}$. pylori infection in gall bladder cancer: clinicopathological study. Tumour Biol. 2015;36(9):7093-8.

41. Jackson SS, Adami HO, Andreotti G, Beane FLE, Gonzalez AB, Buring JE, et al. Associations between reproductive factors and biliary tract cancers in women from the Biliary Tract Cancers Pooling Project. J Hepatol. 2020;73(4):863-72.

42. Andreotti G, Hou L, Gao YT, Brinton LA, Rashid A, Chen J, et al. Reproductive factors and risks of biliary tract cancers and stones: a population-based study in Shanghai, China. Br J Cancer. 2010;102(7):1185-9.

43. Makiuchi T, Sobue T, Kitamura T, Sawada N, Iwasaki M, Sasazuki S, et al. Reproductive factors and gallbladder/bile duct cancer: a population-based cohort study in Japan. Eur $\mathbf{J}$ Cancer Prev. 2017;26(4):292-300.

44. Saranga Bharathi R, Singh R, Gupta R, Verma GR, Kalra N, Kiran $\mathrm{K}$, et al. Female sex hormone receptors in gallbladder cancer. J Gastrointest Cancer. 2015;46(2):143-8. 
45. Arnold M, Pandeya N, Byrnes G, Renehan PAG, Stevens GA, Ezzati PM, et al. Global burden of cancer attributable to high body-mass index in 2012: a population-based study. Lancet Oncol. 2015;16(1):36-46.

46. Bhaskaran K, Douglas I, Forbes H, Santos SI, Leon DA, Smeeth L. Body-mass index and risk of 22 specific cancers: a population-based cohort study of 5.24 million UK adults. Lancet. 2014;384(9945):755-65.

47. Tan W, Gao M, Liu N, Zhang G, Xu T, Cui W. Body Mass Index and Risk of Gallbladder Cancer: Systematic Review and Meta-Analysis of Observational Studies. Nutrients. 2015;7(10):832134.

48. Li L, Gan Y, Li W, Wu C, Lu Z. Overweight, obesity and the risk of gallbladder and extrahepatic bile duct cancers: A meta-analysis of observational studies. Obesity. 2016;24(8):1786-802.

49. Campbell PT, Newton CC, Kitahara CM, Patel AV, Hartge P, Koshiol J, et al. Body Size Indicators and Risk of Gallbladder Cancer: Pooled Analysis of Individual-Level Data from 19 Prospective Cohort Studies. Cancer Epidemiol Biomarkers Prev. 2017;26(4):597-606.

50. Schmidt MA, Marcano BL, Roberts LR. Gallbladder cancer: epidemiology and genetic risk associations. Chin Clin Oncol. 2019;8(4):31.

51. Henley SJ, Weir HK, Jim MA, Watson M, Richardson LC. Gallbladder Cancer Incidence and Mortality, United States 1999-2011. Cancer Epidemiol Biomarkers Prev. 2015;24(9):1319-26.

52. Lorenzo BJ, Boekstegers F, Gonzalez SR, Marcelain K, Baez BP, Barahona PC, et al. Subtypes of Native American ancestry and leading causes of death: Mapuche ancestry-specific associations with gallbladder cancer risk in Chile. PLoS Genet. 2017;13(5):1006756.

53. Jackson SS, Van DWV, Pfeiffer RM, Cook P, Hildesheim A, Pinto LA, et al. Inflammatory profiles in Chilean Mapuche and non-Mapuche women with gallstones at risk of developing gallbladder cancer. Sci Rep. 2021;11(1):3686.

54. Aldouri AQ, Malik HZ, Waytt J, Khan S, Ranganathan K, Kummaraganti S, et al. The risk of gallbladder cancer from polyps in a large multiethnic series. Eur J Surg Oncol. 2009;35(1):48-51.

55. Yagyu K, Kikuchi S, Obata Y, Lin Y, Ishibashi T, Kurosawa M, et al. JACC Study Group. Cigarette smoking, alcohol drinking and the risk of gallbladder cancer death: a prospective cohort study in Japan. Int J Cancer. 2008;122(4):924-9.

56. Lugo A, Peveri G, Gallus S. Should we consider gallbladder cancer a new smoking-related cancer? A comprehensive meta-analysis focused on doseresponse relationships. Int $\mathrm{J}$ Cancer. 2020;146(12):3304-11.

57. Makiuchi T, Sobue T, Kitamura T, Ishihara J, Sawada N, Iwasaki $M$, et al. The relationship between vegetable/fruit consumption and gallbladder/bile duct cancer: A population-based cohort study in Japan. Int $\mathbf{J}$ Cancer. 2017;140(5):1009-19.

58. Larsson SC, Hakansson N, Wolk A. Healthy dietary patterns and incidence of biliary tract and gallbladder cancer in a prospective study of women and men. Eur J Cancer. 2017;70:42-7.

59. Nogueira L, Foerster C, Groopman J, Egner P, Koshiol J, Ferreccio C. Association of aflatoxin with gallbladder cancer in Chile. JAMA. 2015;313(20):2075-7.

60. Koshiol J, Gao YT, Dean M, Egner P, Nepal C, Jones $\mathrm{K}$, et al. Association of Aflatoxin and Gallbladder Cancer. Gastroenterology. 2017;153(2):488-94.

Cite this article as: Tan Z, Deng Z, Gong J. Risk factors for the onset of gallbladder cancer: a review. Int Surg J 2021;8:1951-8. 